\title{
The goal of locomotion: Separating the fundamental task from the mechanisms that accomplish it
}

\author{
James L. Croft ${ }^{1}$ - Ryan T. Schroeder ${ }^{2}$ - John E. A. Bertram ${ }^{1,2,3}$
}

Published online: 13 January 2017

(C) Psychonomic Society, Inc. 2017

\begin{abstract}
Human locomotion has been well described but is still not well understood. This is largely true because the observable aspects of locomotion - neuromuscular activity that generates forces and motions - relate to both the task solution and the problem being solved. Identifying the fundamental task achieved in locomotion makes it possible to critically evaluate the motor control strategy used to accomplish the task goal. We contend that the readily observed movements and activities of locomotion should be considered mechanism(s). Our proposal is that the fundamental task of walking and running is analogous to flight, and should be defined in terms of the interaction of the individual's mass with the medium in which it moves: a low-density fluid for flight, or the supporting substrate for legged locomotion. A rigorous definition of the fundamental task can help identify the constraints and opportunities that influence its solution and guide the selection of appropriate mechanisms to accomplish the task effectively. The results from robotics-based modeling studies have demonstrated how the interaction of the mass and substrate can be optimized, making the goal of movement a defined trajectory of the individual's mass. We assessed these concepts by evaluating the ground reaction forces generated by an optimization model that satisfies the task but uses none
\end{abstract}

James L. Croft

j.croft@ecu.edu.au

1 Centre of Exercise and Sports Science Research, School of Medical and Health Sciences, Edith Cowan University, Perth, Western Australia

2 Biomedical Engineering, University of Calgary, Calgary, Alberta, Canada

3 Cumming School of Medicine, University of Calgary, Calgary, Alberta, Canada of the mechanisms that are available to the human leg. Then we compared this model to normal human walking. Although it is obvious that the specific task of locomotion changes with a variety of movement challenges, clearly identifying the fundamental task of locomotion puts all other features in an interpretable context.

Keywords Locomotion · Walking · Task constraint . Economy

Research into human locomotion has resulted in a thorough understanding of the motions, actions, and control of walking and running. However, it remains unclear why those particular motions and actions are selected by the motor control system. The speed-frequency relationship for human walking is well documented, but why this particular relationship and not another? Likewise, the human walk is generated using a distinctive double-maximum force profile over the contact of each leg-what about this profile makes the normal human walk functionally effective? Even though the ground reaction force profile is generated by the actions of the limb, the coordinated activity involved in human locomotion depends on a centrally generated control strategy. Although much is known about the generation and implementation of locomotion, the reasons why this particular strategy is executed remain obscure, as are the reasons for many of the externally observable effects, such as the ground reaction profile.

The interpretation of motor control strategies and of the mechanisms used to implement them is dependent on a clear understanding of the task being accomplished. In many cases it is reasonably straightforward to define the task and its goal-for instance, with regard to simpler tasks such as reaching, grasping, or manipulating (e.g., Todorov \& Jordan, 2002), coordinating between limbs (e.g., Haken, Kelso, \& 
Bunz, 1985; Kugler \& Turvey, 1987), or even more complex tasks, such as those seen in various sport events, including the high jump (Le Pellec \& Maton, 2000), javelin (Best et al., 1993), baseball pitch (DiGiovine et al., 1992), and golf swing (Nesbit, 2005). Even though walking and running are both important and natural motor tasks, it is not nearly so easy to define precisely what these tasks are, or the goal that the motor control system is accomplishing in each. What if the "task" of locomotion is very different than it obviously appears to be?

Clearly, many task goals of locomotion can vary depending on the situation encountered - for instance, moving up versus down a slope, or over obstacles in an uneven path. These are important aspects of the control and function of locomotion, but they can be viewed as variations originating from the primary, or fundamental, task of locomotion.

What, then, defines the fundamental task of locomotion? Although apparently straightforward, the answer to this question is actually quite elusive. At one end of the spectrum, one might suggest that it is simply moving the individual from one location to another. However, such a general definition does not discriminate between the methods that could be used. For instance, this could serve just as well as the "task" facing a bird that would fly between two locations. It is obvious that legged locomotion and flight are fundamentally different tasks, and so require different task definitions (and different mechanisms are appropriate to accomplish each). Even if one were to restrict the definition to the use of limbs through contact with a substrate, numerous options are available to both humans and animals. For instance, humans can walk, run, hop, or skip (not to mention all the nonupright options, such as crawl, flop, wiggle, etc.). Not surprisingly, the number of discrete gaits available depends on the number of limbs, and is determined by the formula $N_{\mathrm{g}}=(2 n-1)$ ! (where the exclamation indicates the factorial, $N_{\mathrm{g}}$ is the number of discrete gaits available, and $n$ is the number of legs). As a consequence, an upright biped has six possible gaits, a quadruped 5,040, and a sixlegged insect has nearly 40 million. Each of these defined gaits has a near infinite range of gait parameters, such as broad ranges for speed, step length, and frequency. A general description of the task as simply requiring movement from one location to another does not have the precision to be useful in evaluating the strategy the motor control system utilizes to accomplish the task.

Alternatively, one might define the task of human locomotion in terms of the muscle activity patterns, dynamic joint torques, and the sequential foot-ground force applications that characterize normal walking. Again, such a definition is deficient because it overspecifies the "task," in that it implies a solution, rather than the problem that needs to be overcome (Bertram, 2013). In like manner, the task of walking could be defined on the basis of kinematics - the motions that are observed. For instance, Sparrow and Newell (1998) stated,
When the motor response does not involve an implement, such as a tool or a machine, we consider the biomechanical constraints that define an activity as the task. In locomotor activities, such as running, walking, and swimming, the constraints on limb movements that define these activities are the task. For example, walking can be distinguished from running by the constraint that, in walking, one foot must be in contact with the surface at all times. (p. 177)

However, we find such a kinematic definition inadequate for the same reason as the kinetic definition: It describes a solution - what is achieved in normal locomotion-without a clear understanding of "why" those motions occur. If it were not an advantage to have overlap in contacts in normal human walking, it is doubtful that the motor control system would see this as a "constraint." Making the statement: "walking from A to $\mathrm{B}$ requires $\mathrm{X}$ through $\mathrm{Z}$ motions because $\mathrm{X}$ through $\mathrm{Z}$ motions move the individual from $A$ to $B$ " is patently circular, but nonetheless is a trap that is easy to fall into if the task and the current solution the body uses to accomplish the task are not clearly distinguished.

As complex as all aspects of the movement may be, it is possible to define a reaching task in terms of the goalreaching to a particular point, possibly with some applied constraint such as a limited time to achieve the goal. The definition of the task is independent of defining how the task must be completed. Different strategies may be used initially, and the participant may evaluate the errors involved with each of the various strategies available and adjust the control accordingly (Gosselin-Kessiby et al., 2008; Gottlieb et al., 1997). In this way, it is possible to evaluate how the motor control system approaches the task, assesses errors, modifies specific aspects of control through experience, and adapts to perturbations - all of which provide substantial insight into the operational structure responsible for the actions observed. Can the "task" of locomotion be specifically defined independent of the actions that cause it to happen, as has been so useful for investigating a reaching task?

For routine tasks like walking or running, the solution may be so intimately associated with the task that the difference appears obscure. Most of us are not quite so intimately familiar with the locomotory mode of flight. Flight can thus serve as an analogous locomotion form in which it is much less difficult to distinguish task from solution. At its essence, the fundamental task of flight is generating adequate lift to counteract the downward forces of gravity, and adequate thrust to counteract drag forces. This "task" can be achieved through numerous mechanisms. For instance, a fixed cambered airfoil will generate lift if it is moved fast enough via a thrusting engine (as in a standard fixed-wing airplane). Likewise, a rotating blade in a helicopter can be oriented to produce both lift and thrust. Birds accomplish the task through flapping motions of 
a feathered airfoil. Although one might assume that a great deal of understanding would come from knowing the motor control, muscle activities, joint torques, and so forth, in bird flight, these are actually useless for understanding their purpose outside of the context of how the animal interacts with its physical environment. However, once the task of flight is defined (the generation of adequate lift and thrust within a lowdensity fluid), then it is possible to interpret the nuances of physiology (Tucker, 1973), different flight strategies, morphology, and life histories (Pennycuick, 2008). Obviously, layered on top of the fundamental task are other, more specific task goals, such as choice of flight path, rate of progress, and so forth. These higher-level task goals change through time and adapt to the circumstances, giving the appearance that the task of flight is constantly changing. The fundamental task goal of generating sufficient lift and thrust must always be satisfied, however, if flight is to be achieved. Is there a fundamental "task" of human locomotion, comparable to that of flight, that will serve to place the observable mechanisms of locomotion in a functional context?

\section{Artificially solving the challenges of legged locomotion reveals the fundamental task}

Recent robotics modeling studies using much simplified versions of legged locomotion suggest a more satisfactory, and counterintuitive, definition of the "task" with respect to translating a body across a substrate utilizing legs (Garcia et al., 1998; Kuo, 2001, 2002; Srinivasan \& Ruina, 2006). Srinivasan and Ruina, in particular, created a relatively simple computer model composed of a pair of actuated legs supporting a mass (the legs were able to shorten or extend, and the body was simply a point mass located where the legs connected). The model was allowed to determine the most energetically effective way to utilize those legs to move the mass across the supporting substrate. Although simple, this model demonstrated key physical features of the interaction of the system with the surface it moved across, and showed the consequences of using different movement strategies. Despite being able to use its legs in any number of ways, the optimization settled on two robust solutions, one that used an inverted pendular motion as the mass moved over the stance leg, in what is intuitively a walking motion, and one that used an impulsive contact and ballistic flight, paralleling the human running gait (the model also identified a third gait intermediate between walking and running that is not used by humans, but is seen naturally in some ground-dwelling birds; Usherwood, 2010). The inverted pendular gait was the minimum-cost solution for slower progression, whereas the impulse and flight (noncontact) gait was the minimum-cost solution for faster progression.
Bernstein recognized that despite the large number of potential ways that systems with many degrees of freedom can be organized, in locomotion only a few are observed:

Reactive forces during large synergies like running, jumping, or vaulting are so large and variable that they sometimes create virtually unsolvable problems for the coordination of such quick movements....If it were possible to find such a pattern of movement in which the reactive forces did not exert their destructive abilities, the central nervous system would cling to it as to a life belt. Experience shows that only one or two and certainly not more than a few versions of such nonselfdestructable movement patterns remain for large synergies of the type discussed here. These forms are qualitatively very different and are separated by wide intervals filled with impossible patterns. (Latash, Turvey, \& Bernstein, 1996, pp. 196-197)

In stark contrast to the "mechanism"-centered issue identified by Latash et al., the simple model of Srinivasan and Ruina (2006) predicts the appropriate solution under the imposed conditions (solid level substrate, Earth's gravity, and a predetermined speed) without consideration of the limitations of the mechanisms that accomplish it; the model does not possess synergies, so it is unable to "cling to it as to a life belt." We submit that properly defining the task of locomotion directs the subset of options not just to avoid destructive options, but also to spontaneously avoid ineffective ones. In so doing, the value of the few options that are chosen (however complex in their own right) can be assessed within their functional context. It is impossible to properly evaluate any apparent synergies without first understanding what they accomplish.

The Srinivasan and Ruina (2006) model provides a number of important insights, but one of particular relevance to the present discussion is what the model utilized as the goal of the optimization, or how it defined the "task" of locomotion. The model was instructed to identify the movement strategy that would allow it to travel on bipedal legs at a given speed across the surface for the least energetic cost. The energetic cost was purely mechanical, in this case, but originated from two sources - direct loss of energy from the system (to the external environment, causing motion to decrease) and work actively generated within actuators of the system (causing motion to increase).

The model identified a typical walking gait for slower speeds and a running gait for faster speeds. For these gaits, it utilized whole-system kinematic and kinetic patterns very reminiscent of human walking and running; although specific differences between the model and human locomotion are observed (e.g., the model does not possess a jointed knee), the general similarity is obvious, especially considering the 
simplicity of the model. Because the model is simple, however, it is possible to analyze the factors that determine these movement strategies as being energetically optimal under the imposed conditions: The leg motions and ground reaction forces are those that guide the trajectory of the center of mass $(\mathrm{CoM})$ in a manner that interacts most effectively with the supporting substrate. From this perspective, the leg motions and actions mediate the interaction of the mass of the individual (simplified as the CoM) and the substrate and manage the CoM trajectory so that it approaches one that is optimum (at least under the circumstances).

To understand what the legs need to accomplish, it is necessary to understand the main influences on the energetic cost of walking. An important, and often neglected, energy loss occurs during the process of transitioning the trajectory of the CoM from a downward fall at the end of the single-leg stance to upward at the beginning of the subsequent single stance on the contralateral leg-the step-to-step transition (Bertram \& Hasaneini, 2013; Donelan et al., 2002; Kuo et al., 2005). This transition occurs because the leg actively alters the path of the CoM, and energy is lost through such discontinuities in the CoM trajectory (Garcia et al., 1998; Kuo, 2002; McGeer, 1990; Ruina et al., 2005; Srinivasan \& Ruina, 2006). The alternate transition, when the CoM shifts from going upward to downward as it passes over the single stance leg, is largely passive. Although energy is temporarily lost in this transition, it is passively replaced by the action of gravity and is well recognized as the pendular portion of invertedpendulum walking (Kuo et al., 2005).

Energy lost from the system in the downward-to-upward transition (or any other portion of the movement in which negative work is performed) must be repaid with mechanical work generated by the leg actuators if a consistent average speed is to be maintained. Positive work from the leg is required, but this can come for two related, but subtly different, reasons. Leg work can directly replace energy lost, or it can be used to implement a movement strategy that decreases the amount of energy lost in the first place. It turns out that some important opportunities (strategies) are available through which increased work at one portion of the gait cycle can reduce net losses of the entire cycle, by substantially reducing the losses that occur at another point in the cycle (Collins et al., 2005; Kuo, 2002; Ruina et al., 2005). See, for instance, the preemptive push-off described below.

Given the extremely limited applied constraints for the Srinivasan and Ruina (2006) model, the optimization selected the CoM trajectory that involved the best compromise between limited energy losses and work that the system had to add with the actuated legs. Note here that the "trajectory" described is not simply the path of the CoM, but also involves the dynamic travel of the CoM, in which changes in velocity (magnitude and direction), including the required forces applied to implement those changes, are critical to the energy state of the system (Lee et al., 2011; Lee et al., 2013).

Interestingly, although it is possible to conceive of a strategy in which no external energy losses occur in walking, the optimized trajectory was not one that eliminated external losses entirely. This is because walking with no external loss requires a greater investment of work, through the leg shortening and lengthening over the contact, than is saved by avoiding all loss, a situation confirmed directly for human walking (Gordon et al., 2009; Ortega \& Farley, 2005; see Fig. 1). The model described an energetically optimal CoM trajectory that allowed the best compromise for moving bipedally under the imposed circumstances (Hasaneini et al., 2013).

Defining the task of locomotion as managing the dynamic trajectory of the CoM (so that energy investment in the gait is optimized) recognizes what is ultimately accomplished, independent of how it is achieved (i.e., independent of the mechanisms utilized). In the context of mammalian locomotion in general, this task has also been described as the phenomenon of locomotion, to distinguish it from the mechanisms that produce it (Bertram, 2016), where the mechanisms involve the motor control strategy and the body component movements they elicit. These produce the overt appearance of locomotion that has distracted attention from recognizing the counterintuitive underlying task. If the task of bipedal locomotion is the management of the optimal CoM trajectory under the given circumstances, then all of the observable features of leg use in locomotion-including the muscle activation patterns, the forces and torques produced, the work accomplished, and the energy expended - are the mechanisms responsible for achieving that task. The mechanisms of locomotion are available for the motor control system to manage, in order to successfully achieve the goal: that is, the task of locomotion. Together, the task and mechanisms determine the options available for how locomotion can proceed or be implemented, each of these having their own internal and external limitations and opportunities.

Of course, optimizing energetic investment is not the only factor that could determine an optimum gait. Myriad influences, ranging from maintaining stability (Cooper et al., 2008) to sending signals about health and emotional state (Buchner et al., 1996; Schneider et al., 2014), will have some effect on the final optimization solution selected under a given circumstance. However, in most common situations, including the standardized circumstances of a laboratory investigation of walking or running, energetics will be a dominating contributor to the task faced by the individual, and thus will be a critical determiner of the effective optimization strategy. Without understanding the role energetic optimization plays in determining the solution set available to the motor control system, it would likely be impossible to properly identify the effects of other influences and the ever-changing rank order of priority influence that is the challenge of understanding 


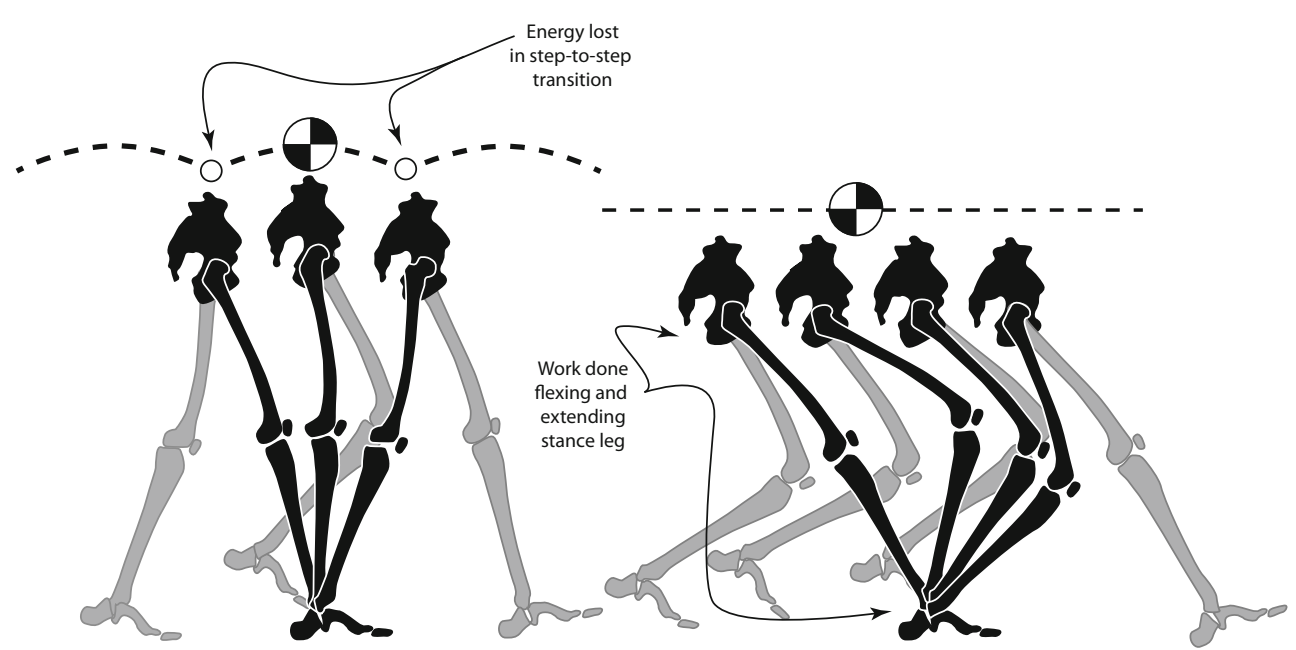

Fig. 1 Illustration of the major sources of mechanical energy cost in walking. At left is normal walking, emphasizing the loss of energy during the step-to-step transition. At right is walking without variation in CoM height (eliminating the abrupt step-to-step transition loss). For this type of walking, the cost of leg work increases beyond the effort saved by eliminating the losses realistic optimization strategies. Recognition of this hierarchy provides a new perspective on the control process and suggests novel routes to systematically isolate and evaluate contributing influences that affect the motor control strategy and the central nervous system's selection processes.

At this point, it is likely of value to clarify our use of the concept "optimization." We utilize this term with regard to work-based models that anticipate some key features of human locomotion. We expect, then, that the factors that direct the model to this discrete optimization solution will also influence the movement strategies available for the central nervous system to select from. Human locomotion will not be so discretely optimized, partly because so many competing factors are involved in the complex human system, and also partly because there are real limitations to its capability to sense optimal strategies, especially in real time. These limitations are often manifest in the time and effort spent evaluating and fine-tuning alternate strategies until the system is satisfied that any additional gains in economy are either nonexistent or negligible - that is, until the solution is optimal.

The task definition described above alters the view originated largely by Bernstein (and continued by most who have followed his work), in which the task in locomotion is more generally defined by the action of what is referred to here as "mechanisms." What might appear to be the specific tasks of applying force and torques at specified stages of the gait, generating work and power that cause the observable motions, should be viewed as the mechanisms through which the task is performed, whereas the task itself (changes in the motion of the CoM), formerly seen largely as a by-product of the activities of the task (Farley \& Ferris, 1998; Lee \& Farley, 1998), has a reversed role in which it serves as the target of those activities. This perspective, which derives naturally from definition of the task in locomotion, puts the mechanisms responsible, including the motor control strategies, in a functional context; what may have been interpreted as the outcome becomes the initial goal.

\section{Disentangling task, mechanism, control, and observed characteristics: Ground reaction forces in human walking as an illustrative example}

One characteristic of normal human walking is the distinctive profile of the ground reaction force (GRF; Fig. 2). It is recognized that these forces arise from the activity of the muscles acting on the musculoskeletal system within the context of the inertial forces and gravitational acceleration acting on the system (Winter, 2009). It is recognized that the motor control system is responsible for generating those muscle activities, and as a consequence, for determining the specifics of the GRF applied by each leg. But does this activity pattern arise internally, as a selected objective that generates "walking," or does the pattern observed occur as the solution required in order to accomplish the task of walking? These alternatives may appear as only subtle versions of each other, but the distinction will have profound influence on how motor control activity is interpreted and how its management is understood.

Consider, as an example, the GRFs of normal human walking. Is the distinctive GRF pattern of the human walk the result of an internally imposed motor generation of the walking gait, or are the options available to the motor control system determined by what those forces must accomplish (and when)? In this section, the purpose of the human walking GRFs is explained, as a means of demonstrating the interdependency of the task definition and the interpretation of the control scheme.

The most straightforward feature of the GRF is the average vertical force magnitude. Since force is the product of the 


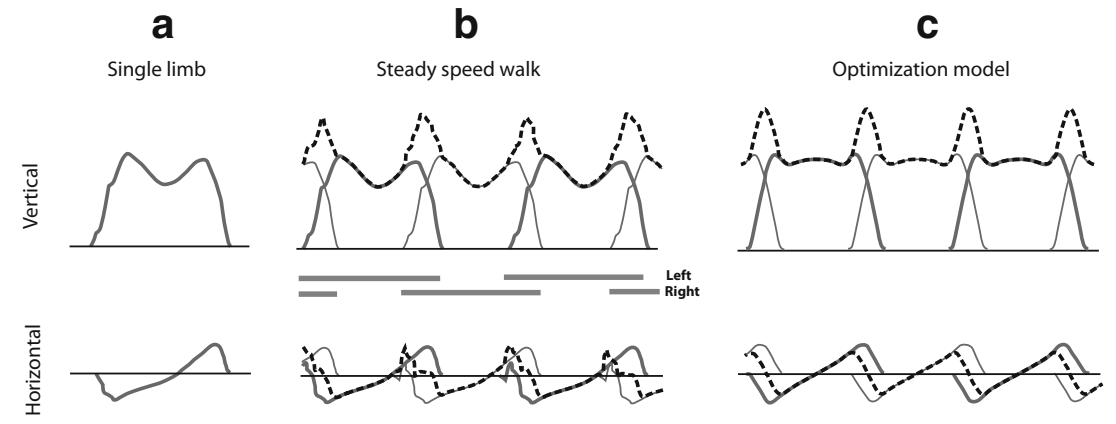

Fig. 2 Ground reaction forces for bipedal walking. (A) Vertical and horizontal ground reaction forces observed over the contact of the foot in normal walking. (B) A series of alternating contralateral contacts in normal walking, showing how the simultaneous push-off and contact forces during double stance combine to produce large vertical forces on the body with relatively modest net horizontal forces. (Total force is shown as a dashed line.) (C) Vertical and horizontal ground reaction forces generated from a simple inverted-pendulum optimization model of bipedal walking. This model optimizes mechanical cost by using the legs to manage the CoM trajectory, but the legs do not use the same mechanisms available to human legs

push-off of the previous stance leg combines with the vaulting action of the next stance leg to complete the CoM directional change over the course of the step-to-step transition (Figs. 3c, d).

During double stance (the transition between support limbs), both legs are applying force simultaneously, resulting in a substantial increase in vertical force on the body (and a substantial decrease in horizontal force; Fig. 2B). This indicates one of the main factors influencing the mechanical cost (and subsequently, the metabolic cost) of walking: the dynamics involved with altering the path of the CoM as it falls forward at the end of one single limb stance, and the actively mediated (by both stance limbs working in an integrated manner) transition that converts the downward (and forward) motion of the CoM to upward (and forward) as the individual progresses through a stride. As we described above, the opposite transition, from upward to downward, occurs passively as a result of the action of gravity, which spontaneously converts kinetic energy to potential and back again as the CoM moves over the strut-like single stance leg. Although it is an important component of the walking stride, single stance involves a relatively low energetic cost, and although an exchange between potential and kinetic energy certainly occurs in this portion of the stride, it is not a particularly critical determinant of the leg use strategy in walking. Instead, the dynamics of the transition between limbs involve the greatest opportunity for energy loss from the system, and so must be managed effectively (relatively precisely). The walking stride involves a low-energetic-cost component, the near passive invertedpendulum transition from upward to downward over a single-leg stance, and a high-cost component, as both legs manage the transition from downward to upward during the step-to-step transition (Kuo et al., 2005; Ruina et al., 2005).

Analysis of the mechanisms of energy loss during the step-to-step transition, even in a very simplified conceptual form, can provide substantial insight into the functional determinants of effective walking (Bertram \& Hasaneini, 


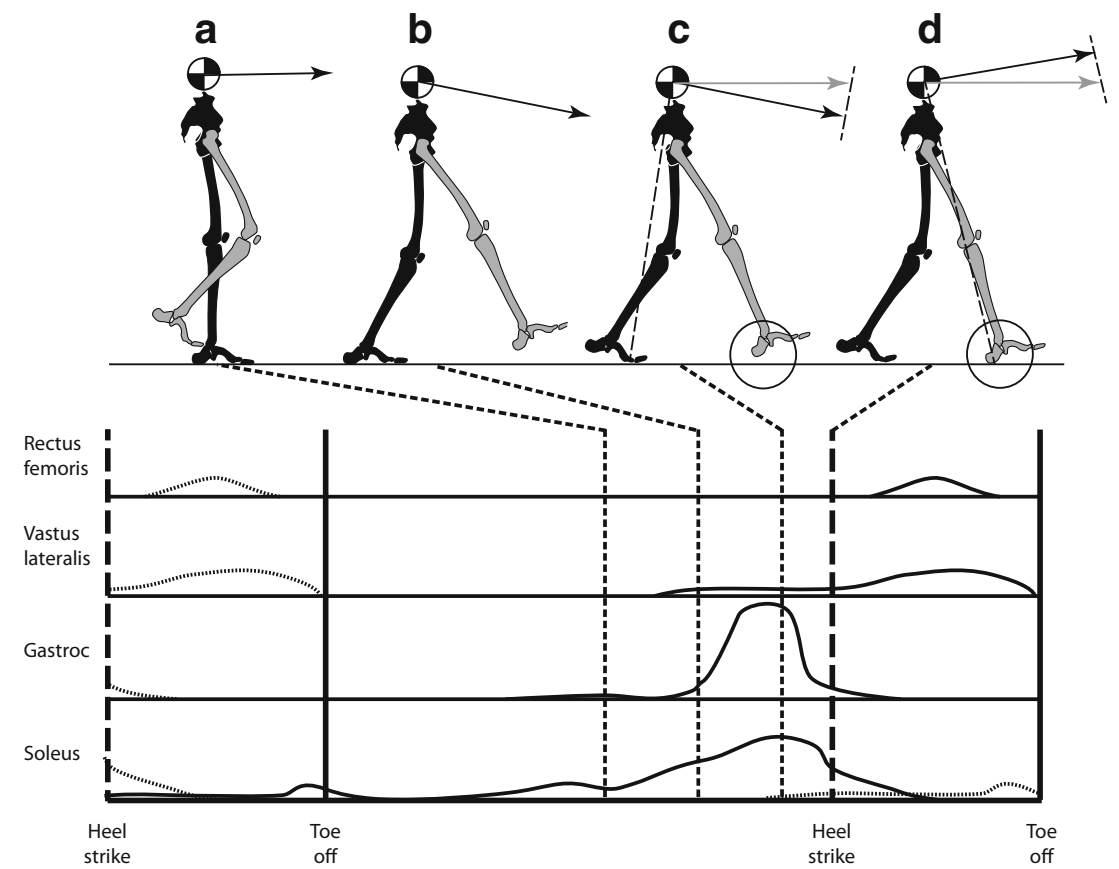

Fig. 3 Key leg muscle activity (knee extensors and foot plantar flexors), relative to CoM velocity changes over the stride. At midstance (a), the leg acts largely as a passive strut, with the CoM velocity oriented horizontally with a modest magnitude, and the CoM passes over the top of the inverted-pendulum arc. CoM velocity magnitude increases as it falls forward (b) in the second half of the arc. Just prior to heel strike (c), gastrocnemius and soleus activity indicates plantar flexing of the foot in the preemptive push-off. This push-off shifts the orientation (and magnitude) of the CoM velocity toward the horizontal. With the leg acting as an extensible strut, the thrust is between the point of contact and the CoM

2013). An abrupt change in direction of a moving mass will result in a transfer of energy between the mass and whatever causes the directional change. This is likely the main reason that the transition from one support leg to the other appears relatively smooth; the alternative involves substantial energy loss. In level walking, the mass is that of the individual, and the change in direction is caused by the interaction of the legs with the supporting substrate. Consider, for example, a spoked wheel (like an old-fashioned wagon wheel) without a rim. As the wheel rolls onto the next spoke, the hub of the wheel (and its mass) will be forced to change direction as it moves over the spoke. Energy will be lost to the system at the point of contact of the spoke, and if the wheel is to keep rolling, energy needs to be added (i.e., work needs to be done to push the wheel forward). ${ }^{1}$ If the spoke that was previously bearing the mass could provide a forward push-off, then the velocity would be increased, to prepare the system to move as an inverted pendulum over the new supporting spoke (which acts simply as a supporting

\footnotetext{
${ }^{1}$ For clarity, it should be noted that this form of energy loss is a completely separate phenomenon from frictional energetic losses that occur when a wheel (even with a rim) slowly rolls to a stop.
}

(dashed line), and this causes a parallel shift in the CoM velocity vector tip. The horizontal orientation of the CoM vector at heel strike (d) means that the transition to the next leg will be smoother than if the preemptive push-off did not take place. The new stance leg also acts like a strut and shifts the CoM velocity vector in a parallel direction. Although energy is lost in this transition, the amount is substantially less than with any other transition strategy. EMG activity of key leg muscles are shown below; solid profiles relate to limb in black, dashed profiles to the contralateral limb (data modified from Cappellini et al., 2006)

strut). However, in this case substantial energy is lost each time the next spoke clunks onto the surface and diverts the fall of the mass. Interestingly, much of this loss can be avoided if the timing of the thrust from the previous support is shifted slightly, and this adjustment of timing turns out to be an important strategy utilized to make normal human walking energetically effective.

If work is added to the rimless wheel just before the next spoke makes contact, the wheel will roll much more effectively. Calculations indicate that only one-third of the work needs to be done if the push comes prior to the next contact rather than following it (Ruina et al., 2005). This situation exists because the relationship between the thrust of the push and the axis of the diverting spoke is altered in a manner that decreases the energy loss over the course of the transition between supports (Bertram \& Hasaneini, 2013; Ruina et al., 2005). The thrust from the previous stance leg prior to heel strike has been termed a "pre-emptive push-off" (Collins et al., 2005).

Although the human leg is much more complex than the rimless wheel, a similar energy-saving technique is possible by adding a forward thrust just prior to the contact of the next support limb (the preemptive push-off). Likewise, 
the contact of the next support limb requires that the new stance limb not buckle but provide a spoke-like (strut-like) support to complete the transition of direction of the CoM from forward and downward to forward and upward. The maxima of the vertical GRF that occur near the beginning and the end of stance for each leg should be viewed with regard to what the contralateral leg is simultaneously doing. The preemptive push-off from the previous stance leg causes the force maximum late in contact, which begins just prior to the alternate leg's contact, and occurs in order to initiate the energetically effective transition to the contralateral limb. Contact and applied load as the new stance leg transitions to a supporting role cause the force maximum early in contact, but this is the second half of the downward-to-upward transition that the mass of the body experiences. These GRFs are precisely timed events critical to the energy effectiveness of the transition (Hasaneini et al., 2013). Even though the GRF of an individual leg has the distinctive double maximum, the force applied to the whole body results from the combined effects of the legs during double stance, so the GRF that affects the CoM has a single maximum occurring during double stance, as support transitions from one leg to the next. The integrated function of the combined legs mediates the CoM trajectory as it transitions from downward to upward for the next portion of the cycle (Fig. 2B).

Several options for the timing of the contact and pushoff could allow for bipedal walking, but only one of these is energetically effective. That is, the motor control system could time the action of each limb differently and still provide stable bipedal motion, albeit for a greater energetic cost. The dynamics of the task dictate the most energetically effective GRF options. It is possible to imagine a variety of ways that the leg could be used to generate forces that could propel the individual forward. For instance, the leg could be used as a lever instead of as a relatively rigid strut, and torques at the hip could be used to generate the required forces. However, modeling of a system like this indicates that such an option requires substantially more energetic investment than does using a preemptive push-off with strut-like legs (Kuo, 2002). In a similar way, the loss of ankle plantar flexion capacity requires that the system find another mechanism for extending the leg to generate the preemptive push-off. For instance, when high-heeled shoes force the foot into a fixed plantar-flexed position (thus preventing it from extending the leg to produce the preemptive push-off), the only joint in the sequence available to compensate appears to be the lower lumbar spine, where tilting of the pelvis effectively extends the support leg relative to the CoM. There is substantial direct evidence that plantar flexor moment is severely reduced when high-heeled shoes are worn, but the GRF profile remains quite similar to low-heeled or unshod walking (Simonsen et al., 2012). Opila-Correia (1990) reported that there was no significant difference in pelvic motion between participants wearing low and high heels; however, speed and step length were reduced in highheeled walking. Equivalent pelvic tilt for shorter steps means that the relative pelvic tilt in the high-heel condition was increased.

The discussion above considers the function of the GRF with regard to the energy-effective transition between supporting legs. How much of the GRF pattern is determined by the specifics of the action generated by the leg (what can be referred to as the "mechanisms" of the leg action), and how much is determined in order to accomplish the task-where the task is managing the interaction between the mass of the body and the substrate, to optimize energetic investment? A simple invertedpendulum optimization model with extensible, telescoping legs supporting a point mass (following Srinivasan \& Ruina, 2006; see Fig. 4) can be used to demonstrate the "whole-body" versus "within-limb" influences. In this model, a stochastic algorithm is used to determine a solution with global optimality in which the model determined the control necessary for minimized mechanical work (an additional penalty for excessive force rate development is required, so as to avoid the model settling on the energetic minimum with unrealistic solutions of infinite impulsive contact forces). The GRF resulting from the optimization solution is shown in Fig. 2C, and details are provided in Appendix A.

Although some differences between the force profile of the model (Fig. 2C) and the normal human walk (Fig. 2B) can be detected, this optimization model selects the essential aspects of the force profile that must be applied to the substrate if the CoM is to travel effectively over the surface on two legs. This force profile is generated in spite of the model not having any of the features normally associated with the function of the human leg in walking - for example, feet, ankles, knees, antagonistic flexor and extensor muscles, complex muscle synergies, and so forth. The similarity between the two GRF patterns indicates that much of the pattern observed in human walking occurs to produce an energetically costeffective solution to the challenge of moving the body mass across the substrate on alternating ground contact points, regardless of the organization and functional details of the "leg." Although the motor control system is responsible for activating the musculoskeletal machinery available to provide these motions/forces, the strategy of activations and internal forces and generated torques cannot be interpreted without understanding the overall task they are recruited to achieve - which is the appropriate CoM trajectory and interaction with the substrate. Recognizing the functional task of bipedal locomotion defines the objective of those motions, and so defines the goal of the activity. 


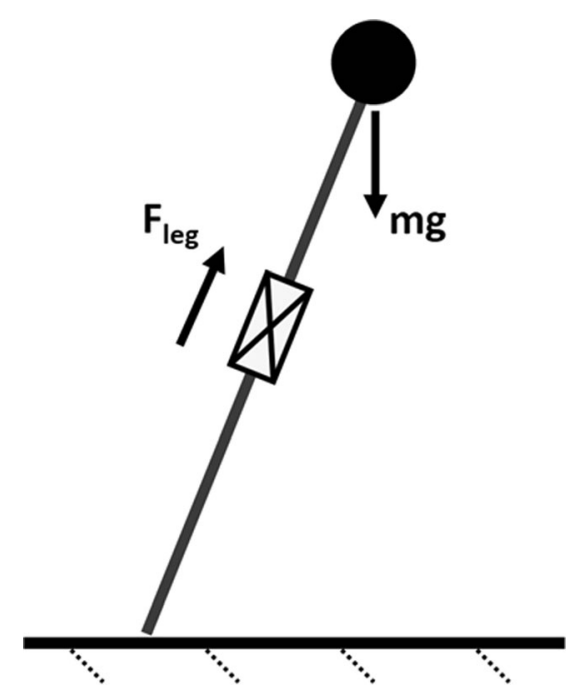

Fig. 4 A reductionist biped model uses massless, telescoping legs to perform work on a point mass, $m$, that represents the body. The acceleration due to gravity is listed with the variable $g$, and the leg force is noted by $F_{\text {leg }}$

If humans operate in a manner similar to the model, then one of the critical constraints acting on the system, which limits the options for the optimization of the CoM trajectory, is energetic cost. If this were not the case, a vast array of alternate solutions could be arrived at, each with its own subset of opportunities and compromises, but none with a better cost effectiveness. Although in our everyday experience we generally have an overabundance of available energy, this was likely not the situation during the evolution of the unique human locomotory system (Pontzer et al., 2012; Sockol et al., 2007). Since even small energetic savings per step can sum to considerable savings over a day/season/lifetime (Bertram, 2016), it may not be surprising that energetics should play such an important role in the task as it is defined for locomotion. This may also explain why the task of locomotion needs to be defined in a different manner than many other movement tasks - particularly than discrete tasks involving the upper extremities (Johansson \& Edin, 1993), which, although complex and critical to effective function and manipulation, do not carry the energetic burden that locomotion does.

To apply this approach to other tasks, one simply has to identify the fundamental features on which function ultimately depends, and then seek to understand how the limb mediates the interaction of the organism with the surface. This sounds easier than it is in practice. For example, in rock climbing, the fundamental task seems to be raising the CoM vertically, but this is similar to defining walking as moving the mass horizontally. Climbers must apply forces through the hand and foot holds that oppose their body mass and also control rotational movement about those holds (Quaine et al., 1997). Whether these constraints define the fundamental task of climbing remains to be determined.
The simplicity of the optimization models used here (Garcia et al., 1998; Kuo, 2002; McGeer, 1990; Srinivasan \& Ruina, 2006) precludes many of the alternate optimizations that could be suggested as explanations of the observed motor control patterns in human walking. For instance, it has been suggested that the preemptive thrust at the end of stance functions to set up the proper starting conditions for the appropriate trajectory of the jointed swing limb, so that the foot does not scuff as it moves into position for the next heel strike (Neptune et al., 2001). The simple model (Fig. 4) displays the preemptive push-off but does not have either a jointed leg (knee) or a foot to scuff. In the complexity of the human leg, however, it is likely that the observed preemptive push-off affects both loss mitigation and swing leg positioning, since both are advantageous and the required activities are not mutually exclusive. However, this indicates two features that are relevant to understanding the task of locomotion. The models do not possess the same mechanisms responsible for motion that human or animal systems do, yet in many regards they predict what those mechanisms must accomplish for the task of locomotion to be achieved. As well, when competing or integrated actions are inevitably involved with the motions, determining the task(s) and the hierarchy of influences allows each action to be placed in context and provides a route through which the influence of each can be experimentally evaluated. With a clearly defined, albeit counterintuitive, task for locomotion, the mechanisms responsible for effective locomotion can be analyzed within a functional context, providing an avenue for interpreting both normal and abnormal locomotion and its control.

Author note Partial funding for this work was provided by the Edith Cowan University Capability Enhancement Scheme.

\section{Appendix A}

Given the two degree-of-freedom system (analogous to Srinivasan and Ruina's, 2006, model), the following equations of motion consider both leg forces and gravitational forces acting on the point-mass (Fig. 4).

$$
\begin{aligned}
& m \ddot{x}=F_{L}(t) *\left[\frac{x-x_{f L}}{L_{L}}\right]+F_{R}(t) *\left[\frac{x-x_{f R}}{L_{R}}\right] \\
& m \ddot{y}=F_{L}(t) *\left[\frac{y}{L_{L}}\right]+F_{R}(t) *\left[\frac{y}{L_{R}}\right]-m g
\end{aligned}
$$

In the equations above, the subscripts $L$ and $R$ indicate the left and right legs, respectively. The variable $L$ is the leg length (constrained by a maximum value of $0.92 \mathrm{~m}$ considered for the model). The quantity $m$ is the point-mass representing the body (a value of $80 \mathrm{~kg}$ was used in this model). $F$ is the extension force of a given leg. The variables $x_{f L}$ and $x_{f R}$ are 
the local horizontal coordinates describing the left and right foot positions, respectively. Finally, $x, \ddot{x}, y$, and $\ddot{y}$ are the horizontal position, the horizontal acceleration, the vertical position, and the vertical velocity, respectively, of the point-mass's local coordinate system.

Solutions to these equations were found by implementing a control optimization technique with software in the MATLAB, MathWorks environment (GPOPS-ii; Patterson \& Rao, 2014). Essentially, this optimization method utilizes an interior-point method to converge upon the model's local minimum for a predefined cost function (the locality of these minima depends on the initial conditions, which are chosen at random; e.g., a stochastic algorithm). After all the local minima are found, only the global optimal solution is considered for analysis. The following expression describes the cost function optimized in this model:

$C=\int_{t_{o}}^{t_{f}}|W|+\varepsilon F^{2} d t$

The equation above considers two cost terms in the integrand: (1) mechanical power, determined by leg force times leg extension velocity, and (2) the force rate squared. Note that the second term is merely a means to deter the model from converging on solutions with unrealistic infinite impulsive forces. The magnitude of this cost term is not easily derived, so a small scaling constant $(\varepsilon)$ was used for weighting.

Only steady-state solutions were considered for moderate walking at $1 \mathrm{~m} / \mathrm{s}$. Step length was enforced as a function of velocity in the form of a model constraint.

\section{References}

Bertram, J. E. A. (2013). Gait as solution, but what is the problem? Exploring cost, economy and compromise in locomotion. Veterinary Journal, 198(Suppl. 1), 3-8.

Bertram, J. E. A. (2016). Understanding mammalian locomotion: Concepts and applications. Hoboken: Wiley-Blackwell.

Bertram, J. E. A., \& Hasaneini, S. J. (2013). Neglected losses and key costs: Tracking the energetics of walking and running. Journal of Experimental Biology, 216, 933-938.

Best, R. J., Bartlett, R. M., \& Morriss, C. J. (1993). A three-dimensional analysis of javelin throwing technique. Journal of Sports Sciences, 11, 315-328.

Buchner, D. M., Cress, M. E., Esselman, P. C., Margherita, A. J., de Lateur, B. J., Campbell, A. J., \& Wagner, E. H. (1996). Factors associated with changes in gait speed in older adults. Journal of Gerontology, 51A, M297-M302.

Cappellini, G., Ivanenko, Y. P., Poppele, R. E., Lacquniti, R. E. (2006). Motor patterns in human walking and running. Journal of Neurophysiology, 95(6): 3426-3437.

Collins, S., Ruina, A., Tedrake, R., \& Wisse, M. (2005). Efficient bipedal robots based on passive-dynamic walkers. Science, 307, 10821085.

Cooper, R. C., Prebeau-Menezes, L., Butcher, M. T., \& Bertram, J. E. A. (2008). Step length and required friction in walking. Gait and Posture, 27, 547-551.
DiGiovine, N. M., Jobe, F. W., Pink, M., \& Perry, J. (1992). An electromyographic analysis of the upper extremity in pitching. Journal of Shoulder and Elbow Surgery, 1, 15-25.

Donelan, J. M., Kram, R., \& Kuo, A. D. (2002). Mechanical work for step-to-step transitions is a major determinat of the metabolic cost of human walking. Journal of Experimental Biology, 205, 3717-3727.

Farley, C. T., \& Ferris, D. P. (1998). Biomechanics of walking and running: center of mass movements to muscle action. Exercise and Sport Sciences Reviews, 26, 253-286.

Garcia, M., Chatterjee, A., \& Ruina, A. (1998). The simplest walking model: Stability, complexity, and scaling. Journal of Biomechanical Engineering, 120, 281-288.

Gordon, K. E., Ferris, D. P., \& Kuo, A. D. (2009). Metabolic and mechanical energy costs of reducing vertical center of mass movement during gait. Archives of Physical Medicine and Rehabilitation, 90(1), 136-144.

Gosselin-Kessiby, N., Messier, J., \& Kalaska, J. F. (2008). Evidence for automatic on-line adjustments of hand orientation during natural reaching movements to stationary targets. Journal of Neurophysiology, 99, 1653-1671.

Gottlieb, G. L., Song, Q., Almeida, G. L., Hong, D., \& Corcos, D. (1997). Directional control of planar human arm movement. Journal of Neurophysiology, 78, 2985-2998.

Haken, H., Kelso, J. A. S., \& Bunz, H. (1985). A theoretical model of phase transitions in human hand movements. Biological Cybernetics, 51, 347-356.

Hasaneini, S. J., McNab, C. J. B., Bertram, J. E. A., \& Leung, H. (2013). The dynamic optimization approach to locomotion dynamics: Human-like gaits from a minimally-constrained biped model. Advances in Robotics, 27, 845-859.

Johansson, R. S., \& Edin, B. B. (1993). Predictive feed-forward sensory control during grasping and manipulation in man. Biomedical Research, 14(Suppl. 4), 95-106.

Kugler, P. N., \& Turvey, M. T. (1987). Information, natural law, and the self-assembly of rhythmic movement. Basingstoke: Routledge.

Kuo, A. D. (2001). A simple model of bipedal walking predicts the preferred speed-step length relationship. Journal of Biomechanical Engineering, 123, 264-269.

Kuo, A. D. (2002). Energetics of actively powered locomotion using the simplest walking model. Journal of Biomechanical Engineering, 124, 113-120.

Kuo, A. D., Donelan, J. M., \& Ruina, A. (2005). Energetic consequences of walking like an inverted pendulum: step-to-step transitions. Exercise and Sport Sciences Reviews, 33, 88-97.

Latash, M. L., Turvey, M. T., \& Bernstein, N. A. (1996). Dexterity and its development. Mahwah: Erlbaum.

Le Pellec, A., \& Maton, B. (2000). Anticipatory postural adjustments depend on final equilibrium and task complexity in vertical high jump movements. Journal of Electromyography and Kinesiology, 10, 171-178.

Lee, D. V., Bertram, J. E. A., Anttonen, J., Ros, I. G., Harris, S. L., \& Biewener, A. A. (2011). A collisional perspective on quadrupedal gait dynamics. Journal of the Royal Society Interface, 8, 1480-1486.

Lee, D. V., Comanescu, T. N., Butcher, M. T., \& Bertram, J. E. A. (2013). A comparative collision-based analysis of human gait. Proceedings of the Royal Society B, 280, 20131779.

Lee, C. R., \& Farley, C. T. (1998). Determinants of the center of mass trajectory in human walking and running. Journal of Experimental Biology, 201, 2935-2944.

McGeer, T. (1990). Passive dynamic walking. International Journal of Robotics Research, 9, 62-82.

Neptune, R. R., Kautz, S. A., \& Zajak, F. E. (2001). Contributions of the individual ankle plantar flexors to support, forward progression and swing initiation during walking. Journal of Biomechanics, 34 , 1387-1398. 
Nesbit, S. M. (2005). A three dimensional kinematic and kinetic study of the golf swing. Journal of Sports Science and Medicine, 4, 499-519.

Opila-Correia, K. A. (1990). Kinematics of high-heeled gait. Archives of Physical Medicine and Rehabilitation, 71, 304-309.

Ortega, J. D., \& Farley, C. T. (2005). Minimizing center of mass vertical movement increases metabolic cost in walking. Journal of Applied Physiology, 99, 2099-2107.

Patterson, M. A., \& Rao, A. V. (2014). GPOPS-II: A MATLAB software for solving multiple-phase optimal control problems using HPadaptive Gaussian quadrature collocation methods and sparse nonlinear programming. ACM Transactions on Mathematical Software, $41,1-37$.

Pennycuick, C. J. (2008). Modelling the flying bird. San Diego: Academic Press.

Pontzer, H., Raichlen, D. A., Wood, B. M., \& Liberman, D. E. (2012). Hunter-gatherer energetics and human obesity. PLOS ONE, 7, e40503. doi:10.1371/journal.pone.0040503

Quaine, F., Martin, L., \& Blanchi, J. P. (1997). The effect of body position and number of supports on wall reaction forces in rock climbing. Journal of Applied Biomechanics, 13, 14-23.

Ruina, A., Bertram, J. E. A., \& Srinivasan, M. (2005). A collisional model of the energetic cost of support work qualitatively explains leg sequencing in walking and galloping, pseudo-elastic leg behavior in running and the walk-to-run transition. Journal of Theoretical Biology, 237, 170-192.

Schneider, S., Christensen, A., Häußinger, F. B., Fallgatter, A. J., Giese, M. A., \& Ehlis, A.-C. (2014). Show me how you walk and I tell you how you feel-A functional near-infrared spectroscopy study on emotion perception based on human gait. NeuroImage, 85, 380 390.

Simonsen, E. B., Svendsen, M. B., Nørreslet, A., Baldvinsson, H. K., Heilskov-Hansen, T., Larsen, P. K., ... Henriksen, M. (2012). Walking on high heels changes muscle activity and the dynamics of human walking significantly. Journal of Applied Biomechanics, $28,20-28$.

Sockol, M. D., Raichlin, D. A., \& Pontzer, H. (2007). Chimpanzee locomotor energetics and the origin of human bipedalism. Proceedings of the National Academy of Sciences, 104, 12265-12269.

Sparrow, W. A., \& Newell, K. M. (1998). Metabolic energy expenditure and the regulation of movement economy. Psychonomic Bulletin \& Review, 5, 173-196.

Srinivasan, M., \& Ruina, A. (2006). Computer optimization of a minimal biped model discovers walking and running. Nature, 439, 72-75.

Todorov, E., \& Jordan, M. I. (2002). Optimal feedback control as a theory of motor coordination. Nature Neuroscience, 5, 1226-1235.

Tucker, V. A. (1973). Bird metabolism during flight: Evaluation of a theory. Journal of Experimental Biology, 58, 689-709.

Usherwood, J. R. (2010). Inverted pendular running: A novel gait predicted by computer optimization is found between walk and run in birds. Biology Letters, 6, 765-768.

Winter, D. A. (2009). Biomechanics and motor control of human movement. New York: Wiley. 